\title{
FINAL STATE INTERACTIONS IN THE PARTON MODEL AND MASSIVE LEPTON PAIR PRODUCTION
}

\author{
F. HENYEY \\ University of Michigan, Ann Arbor, Michigan 48104, USA \\ and \\ R. SAVIT \\ National Accelerator Laboratory, Batavia, Illinois 60510, USA
}

Received 3 June 1974

\begin{abstract}
We show that when final state interactions are added to the parton model (Drell-Yan) formula for massive lepton pair production, the cross sections decreases. Our proof rests on assumptions similar to those made by Landshoff and Polkinghorne and others. Thus, contrary to previous claims, this effect cannot help explain the discrepancy between the parton model predictions for this process and the BNL data.
\end{abstract}

In any attempt to describe deep inelastic phenomena with a parton model, one must ultimately face the problem of final state interactions. These interactions must be considered for two reasons: First, in quarkparton models they are the mechanism by which the final hadronic state can dispose of its isolated quark quantum numbers. Second, regardless of what the partons are, it has never really been clear how these interactions alter the predictions of the parton model. The orthodox view, proposed by Bjorken, Feynman and others [1], is that final state interactions will not affect many of the gross predictions of parton models for example, the shapes of certain multiplicity distributions, cross sections, etc. While this is an appealing possibility, it is ar from obvious.

Recently, Einhorn and Savit [2] derived rigorous upper bounds on parton model predictions for the process $\mathrm{pp} \rightarrow \mu^{+} \mu^{-} \mathrm{X}$. They proved that the colored quark parton model coupled with the Drell-Yan formula for this process [3] isincompatible with the BNL data [4]. The Drell-Yan formula is derived by neglecting final state interactions (involving wee partons) and is represented by the diagram of fig. 1a. Landshoff and Polkinghorne [5] propose that another diagram (fig. $1 \mathrm{~b}$ ) with pomeron exchange between the upper and lower blobs is just as important as fig. 1a

Address after September 1, 1974: CERN, Division TH, CH-1211 Geneva 23, Switzerland.

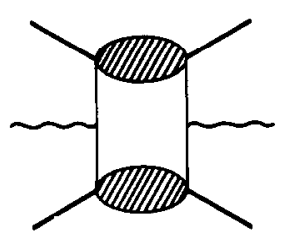

(a)

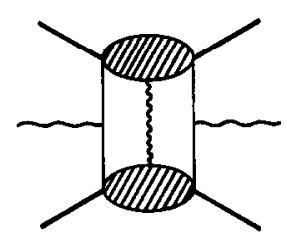

(b)
Fig. 1a. Drell-Yan term for the process $p p \rightarrow \ell^{+} \ell^{-} X$. The lepton pair comes from the decay of the massive photon. 1b. Correction term to fig. 1a. The effects of wee partons are represented by the final state (Pomeron) interaction.

and suggest that it can explain certain features of the BNL data not explained by the Drell-Yan formula. Given the apparent theoretical importance of final state interactions, and the conclusions of ref. [2], a re-examination of the role of final state interactions in the process seems warranted.

In this paper, we will show that according to the usual ideas of hadronic interactions, the LandshoffPolkinghorne diagram, fig. $1 \mathrm{~b}$, cannot explain the discrepancies between the parton model predictions and the data. The reason is that when this diagram is included it lowers the cross section below that predicted by the Drell-Yan term alone, which is already too low to explain the data [2] . Let us first demonstrate this effect. We will then have a number of comments to make. 
The cross section in which we are interested, $Q^{4} \mathrm{~d} \sigma / \mathrm{d} Q^{2}$, is proportional to the imaginary part of the $3 \rightarrow 3$ (proton-proton-photon) forward amplitude. $Q$ is the invariant mass of the muon pair, and according to Drell and Yan [2], this cross section depends only on $\tau=Q^{2} / s$ for nonzero $\tau$. $s$ is the center of mass energy squared of the incident protons. To first order in the final state (pomeron) interaction, we have for this amplitude [6]

$T=B G B^{+}+B G P G B^{+}$.

The two terms on the right correspond to figs. 1a and $\mathrm{b}$, respectively. $B$ is the matrix element which takes the $(\mathrm{pp} \gamma)$ state into the state represented by the two blobs. In the quark parton model, each blob has nonzero triality. For finite (non-zero) $\tau$, each blob has a finite mass, and as $s \rightarrow \infty$ the two blobs are widely separated in rapidity. $G$ is the propagator of the two blob intermediate state, and $P$ represents the final state interaction between the blobs. Since we work only to first order in $P$, we do not need to say whether the $P$ interaction is two blob irreducible or not.

We can write

$$
\begin{aligned}
Q^{4} \mathrm{~d} \sigma / \mathrm{d} Q^{2} \propto \operatorname{Im} T=(1 / 2 \mathrm{i})\left(T-T^{+}\right) \\
\quad=B\left[\operatorname{Im} G+G P \operatorname{Im} G+G \operatorname{Im} P G^{+}+\operatorname{Im} G P^{+} G^{+}\right] B^{+} \\
\quad=B[\operatorname{Im} G-\operatorname{Im} G \operatorname{Im} P \operatorname{Im} G \\
\quad+\operatorname{Im} G \operatorname{Re} P \operatorname{Re} G+\operatorname{Re} G \operatorname{Im}(P G)] B^{+} .
\end{aligned}
$$

Now, $\operatorname{Im} P$ should be a positive quantity since, under the usual assumptions about strong damping in the transverse momentum, the blobs will rescatter primarily in the forward direction where $\operatorname{Im} P$ is positive. Furthermore, we expect $P$ to be primarily imaginary, since at high $s$ and fixed $\tau$ its largest contributor should be the pomeron. This can also be seen by remembering that the pomeron is just a way of describing the wee parton interactions. The only way in which the two blob state differs from a two proton state is that each blob has one less hard parton than the proton from which it came. But the pomeron depends only on wee partons and so the nature of the singularity should not be affected by the removal of one hard parton from each side. (Of course, the magnitude of the coupling of the pomeron to the blots may be affected by the missing partons). To complete our argument, we also need the condition that $(\operatorname{Re} G)^{2} \ll(\operatorname{Im} G)^{2}$. This is a reasonable thing to expect if partons have some finite mass (on the order of a GeV).

Under these assumptions, we can neglect the last two terms in eq. (2), and we have

$Q^{4} \mathrm{~d} \sigma / \mathrm{d} Q^{2} \propto \operatorname{Im} T=B[\operatorname{Im} G-\operatorname{Im} G \operatorname{Im} P \operatorname{Im} G] B^{+}$.

Thus the inclusion of graph fig. $1 \mathrm{~b}$ decreases the cross section.

This argument is quite analogous to the arguments posed many times before about the sign of the two pomeron cuts, and, in general, about the sign of absorptive corrections to high energy processes [e.g. 7]. As in those cases, the minus sign arises from the cuts made to the left and right of the center of the diagram. However, unlike the usual multiperipheral arguments for the case of the two pomeron cut, the negative contributions in this case come from intermediate states with a large rapidity gap i.e., cutting to the left and right of the $P$ interaction of fig. $1 \mathrm{~b}$.

Of course, there are ways to circumvent this argument. Since very little is known about the propagation and strong interactions of systems with nonzero triality it is possible that $\operatorname{Re} G$ and/or $\operatorname{Re} P$ is large, or that $\operatorname{Im} P$ is not positive. Nevertheless, it is in the spirit of many parton models (especially the covariant parton model [5]) that such abnormal behavior does not occur, so that eq. (3) is valid.

There are a number of comments to be made about this result. First, such an additional term cannot fix up the Drell-Yan formula to agree with the data in the scaling region. As shown in ref. [2] the upper bound derived by assuming that only fig. 1 a contributes falls well below the Brookhaven data. Including fig. 1b only decreases the upper bound still further. Notice, in particular, that this means that this diagram cannot explain the shoulder in the data at $Q \sim 3-4$, as has been suggested by Fidler [8].

Second, we want to comment on the region $\tau$ near zero. The arguments which we have presented are certainly valid for finite $\tau$. However, as $\tau \rightarrow 0$, the invariant mass of at least one of the blobs gets large (as a power of $s$ ). This means that, in some sense, the blob may not be close to its "mass shell" in the important region of integration, and so $G$ might no longer be dominated by its imaginary part. Consequently, no 
firm conclusions can be drawn about relative signs of figs. (1a) and (1b) in the limit $\tau \rightarrow 0$.

Third, we remind the reader that it is not clear how to incorporate final state interactions into deep inelastic processes, since, as Landshoff and Polkinghorne point out, their analyticity properties may be quite different [9]. Hence, graphs analogous to fig. 1b might not possess the non-planar topologies which are important for the validity of the argument presented here.

Finally, if the main assumptions of this picture are valid, and if we are allowed to consider only the two terms of fig. 1 , we can make a prediction for the energy dependence and deviations from scaling of this process at higher energies: If the pomeron is a moving pole with intercept 1 , then at fixed $\tau$ the cross section should increase with $s$, because the second term (fig. 1b) will decrease like an inverse power of log s relative the first term. This will be an interesting signal to look for at NAL.

In this paper we have shown that if we apply the usual assumptions of hadronic scattering and propagation to states of nonzero triality, the diagram suggested by Landshoff and Polkinghorne, fig. 1b, decreases, rather than increases the cross section in the scaling region. Hence, it cannot explain the discrepancy between the BNL data the predictions of the DrellYan formula. Moreover, including this diagram decreases the upper bounds of ref. [2], and makes their violation by the data even more striking. We must therefore look deeper in order to understand why the naive quark parton model which has been successfully applied to space-like reactions fails to describe even the general properties of time-like $Q^{2}$ processes.
One of us (RS) would like to thank

R. Blankenbecler, M.B. Einhorn and A.R. White for interesting discussions.

\section{References}

[1] Among the many places this assumption is used or discussed are J.D. Bjorken, Phys. Rev. D7 (1973) 282;

S.M. Berman, J.D. Bjorken and J.B. Kogut, Phys. Rev. D4 (1971) 3388;

R.P. Feynman, Photon-Hadron Interactions (W.A. Benjamin, Inc., 1972);

R. Savit, Phys. Rev. D8 (1973) 274;

R.N. Cahn, J.W. Cleymans and E.W. Colglazier, Phys. Lett. 43B (1973) 323 and ref. [3], below.

[2] M.B. Einhorn and R. Savit, NAL-Pub-74/35-THY, and NAL-Pub-74/41-THY, submitted to Phys. Rev.

[3] S.D. Drell and T.M. Yan, Phys. Rev. Lett. 25 (1970) 316; Ann. Phys. 66 (1971) 578.

[4] J.H. Christenson et al., Phys. Rev. D8 (1973) 2016.

[5] P.V. Landshoff and J.C. Polkinghorne, Nucl. Phys. B33 (1971) 221; Nucl. Phys. B36 (1972) 642.

[6] The symbolic notation we use here follows that used by R. Blankenbecler, SLAC-TN-72-13 (unpublished) and Phys. Rev. Lett. 31 (1973) 964.

[7] I.G. Halliday and C.T. Sachrajda, Imperial College Preprint ICTP 73/18 (1973);

L. Caneschi, Phys. Rev. Lett. 23 (1969) 254;

J.W. Dash, J.R. Fulco and A. Pignotti, Phys. Rev. D1 (1970) 3164;

T.L. Neff, Berkeley preprint LBL 1544 (1973);

A.R. White, Eighth Rencontre de Moriond, Vol. II, ed., J. Tran Than Van, p. 105 (1973); and ref. [6], above.

[8] R.W. Fidler, Phys. Lett. 46B (1973) 455.

[9] P.V. Landshoff and J.C. Polkinghorne, Physics Reports 5C, No. 1 (1972), and references therein. 